\title{
Quantitative Microdialysis: Experimental Protocol and Software for Small Molecule Protein Affinity Determination and for Exclusion of Compounds with Poor Physicochemical Properties
}

\author{
Steven Shave ${ }^{1,+}\left(\mathbb{D}\right.$, Nhan T. Pham ${ }^{1,+}$, Connor B. Śmieja ${ }^{1}$ (D) and Manfred Auer ${ }^{1,2, *(\mathbb{D})}$ \\ 1 School of Biological Sciences, IQB3, University of Edinburgh, The King's Buildings, Max Born Crescent, \\ CH Waddington Building, Edinburgh, Scotland EH9 3BF, UK; s.shave@ed.ac.uk (S.S.); \\ Nhan.Pham@ed.ac.uk (N.T.P.); C.B.Smieja@sms.ed.ac.uk (C.B.Ś.) \\ 2 School of Biological Sciences and Edinburgh Medical School: Biomedical Sciences, University of Edinburgh, \\ The King's Buildings, Edinburgh EH9 3BF, UK \\ * Correspondence: manfred.auer@ed.ac.uk \\ + Both authors contributed equally to this work.
}

Received: 29 June 2020; Accepted: 21 July 2020; Published: 30 July 2020

check for updates

\begin{abstract}
Quantitative microdialysis is a traditional biophysical affinity determination technique. In the development of the detailed experimental protocol presented, we used commercially available equipment, rapid equilibrium dialysis (RED) devices (ThermoFisher Scientific), which means that it is open to most laboratories. The target protein and test compound are incubated in a chamber partitioned to allow only small molecules to transition to a larger reservoir chamber, then reversed-phase high performance liquid chromatography (RP-HPLC) or liquid chromatography-mass spectrometry (LC-MS) is used to determine the abundance of compound in each chamber. A higher compound concentration measured in the chamber that contains the target protein indicates binding. As a novel, and differentiating contribution, we present a protocol for mathematical analysis of experimental data. We provide the equations and the software to yield dissociation constants for the test compound-target protein complex up to $0.5 \mathrm{mM} \mathrm{K}$, and we quantitatively discuss the limitations of affinities in relation to measured compound concentrations.
\end{abstract}

Keywords: label free screening; affinity determination; dialysis; $\mathrm{K}_{\mathrm{D}}$ determination; promiscuity; aggregation; nonspecific binding; protein binding analysis; chromatography; drug discovery

\section{Introduction}

Quantitative microdialysis $(q \mu \mathrm{D})$ is a traditional, yet powerful technique for the analysis of two important properties of small molecules of interest [1-4]: (a) the diffusability of small molecules, which is affected by their intramolecular aggregation and their surface binding properties, and (b) the determination of their affinities to target proteins, using equipment found in many laboratories. Conceptually, dissolved test compounds may freely move between two chambers partitioned by a semipermeable membrane that allows only small molecules to pass through. Along with these freely moving compounds, one chamber also contains either the target protein (to which it is assumed or known to bind to) or one or more control proteins (which serve to test the selectivity of the target protein binding reaction). A thermodynamic equilibrium reached in the absence of a protein after a certain incubation time should result in equal concentrations of free compound in the two chambers. If the compound engages in nonspecific binding towards the material of the chambers or the separating membrane, different compound concentrations will be detected between the two chambers, using any 
of the suitable analysis techniques. In the presence of a target protein binding to the test compound, the two chambers are also expected to contain different compound concentrations (see Scheme 1).

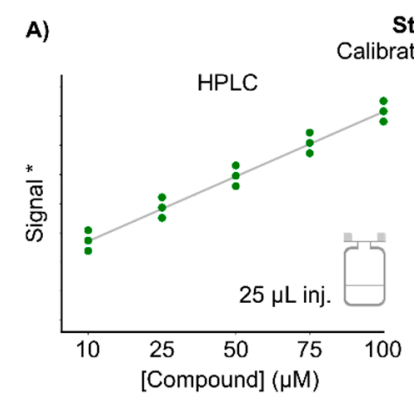

Step 1

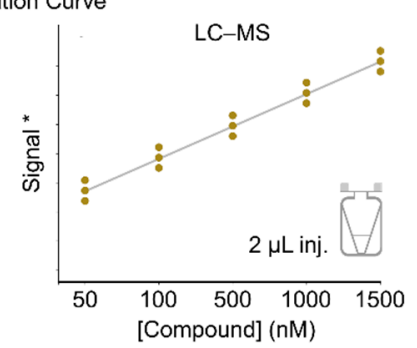

C)
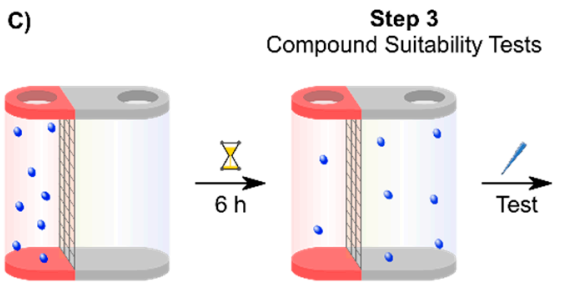

Step 4

Target Binding Tests
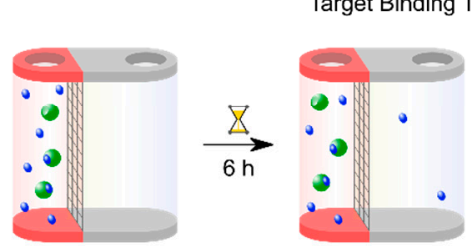

Off-Target Binding Tests
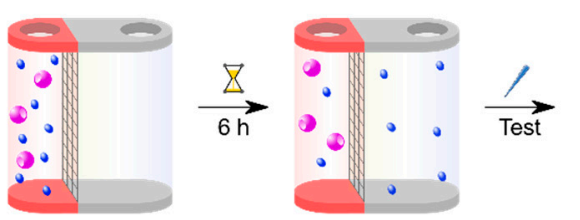

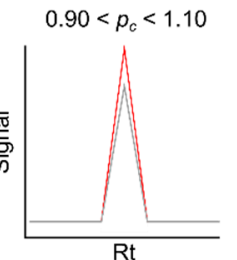

$1.70<p_{t}<5.00$
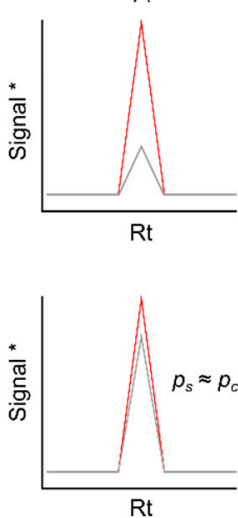

B) Step 2
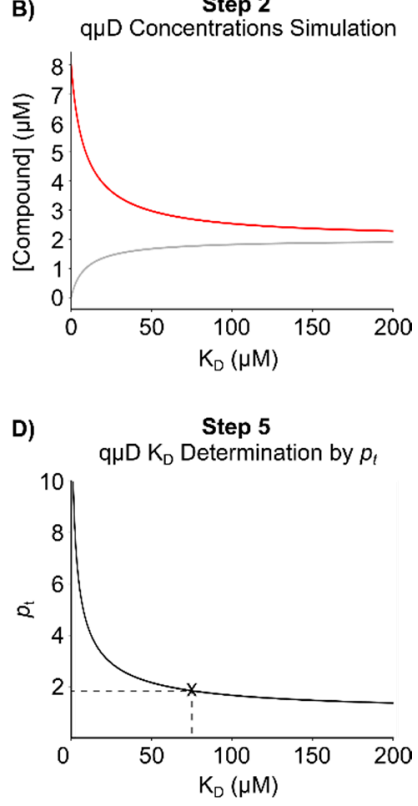

D)

Legend

- Red chamber

- White chamber

- Small molecules

- Target protein

- BSA

NN Cellulose membrane

* For HPLC: absorption at $260 \mathrm{~nm}$ or relative fluorescence intensity For LC-MS: total ion count

Scheme 1. Schematic representation of the quantitative microdialysis (quD) process. Step 1-Determination of the detection sensitivity of test compounds by reversed-phase high performance liquid chromatography (RP-HPLC). Small molecules are monitored either by absorption at $260 \mathrm{~nm}$, or by relative fluorescence intensity at the appropriate, predetermined excitation or emission wavelengths. If LC-MS is used for quantification then the ionisation mode and sensitivity is monitored, and the small molecule peak is resolved by the total ion count. Step 2-Identification of the appropriate target protein concentration. Knowing detection limits and compound solubility, response (compound concentration) can be simulated over a range of $\mathrm{K}_{\mathrm{D}}$ s to ensure detection is possible at the desired $K_{\mathrm{D}}$ range. Step 3-A compound-only experiment is performed to test whether the small molecule is suitable for a dialysis experiment at all. The partition coefficient of the small molecule in the absence of protein ( $p_{c}$, where the ' $c$ ' denotes 'control') needs to be between 0.9 and 1.1. If the partition coefficient is higher, the compound is suggested to be excluded. Step 4: The identical experiment as in Step 3 is repeated, including the target and control proteins in concentrations simulated and predetermined in Step 2. Bovine serum albumin (BSA) is given as an example. RP-HPLC or LC-MS analyses now lead to the partition coefficients both in the presence of the target protein and off-target proteins $\left(p_{t}\right.$, where the ' $\mathrm{t}$ ' denotes the 'target', and $p_{s}$, where the ' $\mathrm{s}$ ' denotes 'selectivity'). Step 5: determining the $\mathrm{K}_{\mathrm{D}}$ of the target-ligand complex using concentrations or $p_{t}$ values. 
Analysis of the compound concentrations in each chamber by RP-HPLC or LC-MS will reveal a higher abundance of the compound present in the protein-containing chamber if the compound binds favourably to that protein. The signal detected represents the sum of the protein-bound compound, and free compound. From these concentrations, the amount of compound bound to the protein can be deduced. Then, since the total amount of protein is known, the binding affinity of the complex can be calculated. Many highly sophisticated techniques have been developed during the last decades to analyse and quantify the interactions of small molecules with proteins, including multiple modes of fluorescence spectroscopy [5], nuclear magnetic resonance (NMR) [6], mass spectrometry [7], affinity selection [8,9] and size exclusion chromatography [10], just to name a few. We apply all of the above in our hit and lead identification projects. The power of the $q \mu \mathrm{D}$ technique lies in its applicability to unlabelled and labelled compounds and proteins, the ease with which it can be set up, and its cost effectiveness. Very importantly, a substantial amount more about the properties of the small molecules can be revealed compared to other techniques, and very low affinity interactions up to $\mathrm{K}_{\mathrm{D}} \mathrm{s}$ of $500 \mu \mathrm{M}$ can be determined reliably. These advantages make $\mathrm{q} \mu \mathrm{D}$ an integral component of our label-free affinity screening platform (LFAP) [11], which is also compatible with fragment-based screening and quantification. Here, we present the experimental and computational design of the $\mathrm{q} \mu \mathrm{D}$ process and provide a detailed protocol and standard operating procedure (SOP) for using our software for $\mathrm{K}_{\mathrm{D}}$ calculation.

\section{Experimental and Computational Design}

\subsection{Experimental Design}

Step 1 of our $q \mu \mathrm{D}$ process is the determination of the appropriate concentration of the small molecules needed for detection. Non-fluorescent or fluorescently labelled compounds can be analysed by RP-HPLC. For non-labelled compounds, a concentration series of 10, 25, 50, 75 and $100 \mu \mathrm{M}$ is recommended, with $25-50 \mu \mathrm{M}$ usually falling in the middle of the linear range. Our lab used this method to confirm hit compounds arising from on-bead screening [12]. Standard library compounds or fragments that do not contain aromatic components cannot be detected by RP-HPLC in sufficiently low concentrations for the $q \mu \mathrm{D}$ process. Instead, these compounds were analysed by LC-MS. To find the appropriate concentration in this case, it must first be established whether the compounds ionise at all, and in which detection mode. The LC-MS method for concentration determination was detailed in Section 3. This protocol was established for analysis by a Thermo Fisher ${ }^{\mathrm{TM}}$ LTQ Orbitrap XL ${ }^{\mathrm{TM}}$. Adaptation of the protocol to other mass spectrometry instruments should be straightforward for any lab proficient in mass spectrometry. In Scheme 1 (Step 1), the concentration range comprising of 50, 100, 500, 1000 and $1500 \mathrm{nM}$, with 250-500 $\mathrm{nM}$ being in the middle of the linear range for most compounds, was derived from an Orbitrap instrument. Step 2 of the $q \mu \mathrm{D}$ analysis process involves simulating the dialysis process. This will inform the experimentalist as to the most suitable concentration of the target protein, to maximise the disparity of compound concentrations between the two chambers, within the detection limits. Therefore, this step is critical to maximise the accuracy of the method. Step 2 was described in more detail in Section 2.2. Step 3 involves testing whether the compound equilibrates uniformly enough between the two dialysis chambers in the absence of the protein, to make it suitable for incubation with the target protein and one or more negative control proteins in Step 4, to test the compounds potential for unselective or nonspecific binding. It is well known in biochemistry that proteins need to be tested for their folding state and aggregation potential. On the other hand, compounds contained in screening libraries, and-more importantly-primary screening hits, are not as routinely investigated for inherent problematic behaviours. These behaviours often lead to false positive and false negative results. Dialysis offers a versatile way to address this; it can be used to test surface binding as a function of buffer composition, $\mathrm{pH}$, additives and other assay variables, as well as to identify dynamic aggregation and promiscuous binding as a function of concentration. Using the predefined compound concentration, $p_{c}$ is determined. This should ideally be below 1.10 after 
an appropriate time period in the target incubation step. Now, having simulated the concentration of the target protein, and proven the purity and suitability of the test compound, Step 4 of the q $\mu \mathrm{D}$ process may be performed. In Scheme 1 (Step 4), a single incubation experiment with the target protein and one with BSA as a control is shown. Additional selectivity and specificity controls can be integrated as needed. The time frame for equilibration can vary as a function of the concentration of the compounds, affinity to the target or temperature, however a study by Walters [13] demonstrates equilibrium of 18 diverse drugs covering a wide range of physicochemical properties is achieved within $6 \mathrm{~h}$. Similar studies by van Liempd et al. [14] and Ye et al. [15] further demonstrate $6 \mathrm{~h}$ to be an adequate equilibration time. We started with $6 \mathrm{~h}$ of incubation, defined by the compound-only experiment. To determine the partition coefficients, the compound concentration needs to be analysed in both compartments. A high concentration of protein can interfere with the chromatographic separation, making it desirable to remove the protein prior to analysis. To this end, methods for protein denaturation and precipitation using organic solvents, acid or heat have been developed [13]. However, we have found that coprecipitation of the compound with the target protein may occur to a certain extent, in line with observations by Vanholder et al. [16], reducing the reliability of the subsequent measurement. Furthermore, adding the denaturation agent to the sample results in a large volume increase, which need to be offset and the original volume established to perform a quantitative analysis. Hence, we present here a method where no protein precipitation or chromatography of the protein containing samples is needed to analyse samples by RP-HPLC. In addition, for the MS analysis, only a simple dilution is required (due to the sensitivity of the mass spectrometer used), which does not compromise the sample quality. At this dilution it was found that the amount of protein present was compatible with our LC-MS setup and therefore analyses could occur without interfering with the compound detection.

\subsection{Materials}

- $\quad$ Self-sticking plate seal (Sigma-Aldrich, Cat no.: Z369667-100EA);

- Rapid equilibrium dialysis (RED) device reusable base plate (Thermo Fisher Scientific, Cat. no.: 89811);

- $\quad$ RED device inserts, 8.0 kDa (Thermo Fisher Scientific, Cat. no.: 89809);

- RED device insert removal tool (Thermo Fisher Scientific, Cat. no.: 89812), or a pair of non-pointy tweezers;

- Assay buffer. A buffer that is compatible with the target protein;

- $20 \%$ ethanol. Made from ethanol (Fisher Scientific, product code 10437341 , purity $\geq 99.8 \%$ ) and Milli Q water;

- $\quad$ Pluronic F127, low UV absorbance, (Thermo Fisher Scientific, Cat. no.: P6867);

- Dimethyl sulfoxide (DMSO) anhydrous, (Sigma Aldrich, Cat. no: 276855, purity $\geq 99.9 \%$;

- $\quad$ Formic acid (FA) for LCMS LiChropur, (Sigma Aldrich, Cat no.: 5.33002, purity 98-100\%);

- HPLC grade $\mathrm{H}_{2} \mathrm{O}$ and Acetonitrile. $\mathrm{H}_{2} \mathrm{O}$ : Milli $\mathrm{Q}$ water with a resistivity $\geq 18 \mathrm{M} \Omega \mathrm{cm}$. Acetonitrile HPLC Plus (Sigma Aldrich, Cat. no.: 34998, purity $\geq 99.9 \%$ );

- Trifluoroacetic acid (TFA) for HPLC, (Sigma Aldrich, Cat. no.: 302031, purity $\geq 99.0 \%$ );

- $1.5 \mathrm{~mL}$ Eppendorf safe-lock tubes (Eppendorf, Cat. no.: 0030120086) or $1 \mathrm{~mL}$ glass vials (VWR, Cat no.: 548-0385, and lids, Cat. no.: 548-0389);

- Chromacol glass insert vials, (VWR, Cat. no.:548-0260);

- Insert vial snap caps (VWR, Cat. no.:548-0265);

- Standard laboratory orbital shaker, such as Ika Schüttler MTS 4 S 2 Microtiter Shaker.

All reagents were used as received. 


\subsection{Equipment}

- Agilent 1100 series HPLC system

RP-HPLC method: RP-HPLC analysis were performed on an Agilent 1100 series HPLC system, consisting of a quaternary pump (G1311A), a degasser (G1322A), a well plate autosampler (G1367A), a fluorescence (FLD) detector (G1321A) and a diode array (DAD) detector (G1315B). The column used was an Agilent ZORBAX SB-C18, $4.6 \mathrm{~mm} \times 150 \mathrm{~mm}, 3.5 \mu \mathrm{m}$ particle diameter and $80 \AA$ pore size. Analyses were performed using a gradient of solvent $\mathrm{A}, \mathrm{H}_{2} \mathrm{O}$ containing $0.1 \%$ trifluoroacetic acid (TFA) and $\mathrm{B}$, Acetonitrile (MeCN; HPLC grade) containing $0.1 \%$ TFA with a flow rate of $0.8 \mathrm{~mL} / \mathrm{min}$. The gradient used: 0-5 min, 5\% B; 5-24 min, 5-100\% B, 24-26 min, 100\% B; 26-27 min, 100-5\% B and 27-30 min, 5\% B. HPLC traces were always measured at absorptions of $210 \mathrm{~nm}, 220 \mathrm{~nm}, 260 \mathrm{~nm}$ and $280 \mathrm{~nm}$. All data analyses were performed using the Agilent Chemstation software v.03.01.

- $\quad$ Thermo Fisher ${ }^{\mathrm{TM}}$ LTQ Orbitrap XL ${ }^{\mathrm{TM}}$.

- Waters Acquity UPLC ${ }^{\mathrm{TM}}$ system.

LC-MS method: All LC-MS analyses were performed on a Waters Acquity UPLC system consisting of a cooled sample manager and a binary solvent manager coupled to the electrospray ionisation (ESI) source of the LTQ Orbitrap via an in-house filled column, $0.2 \mathrm{~mm} \times 120 \mathrm{~mm}, 5 \mu \mathrm{m}$ particle diameter and $120 \AA$ pore size (ReproSil-Pur 120 C18-AQ, $5 \mu \mathrm{m}$ ). The solvent system for elution was: A- $\mathrm{H}_{2} \mathrm{O}$ containing $0.1 \%$ formic acid and $\mathrm{B}-\mathrm{MeCN}$ containing $0.1 \%$ formic acid. A standard gradient method was used for analysis, which was: 0-3 min, 0.5\% B; 3-18 min, 70 \% B; 18-22 min, 95\% B and 22-25 min $0.5 \% \mathrm{~B}$; with a flow rate of $8 \mu \mathrm{L} / \mathrm{min}$. The mass analyser was an LTQ Orbitrap (Thermo Finnigan) with an ESI injection and it was set to record mass data at the start of the gradient. Thermo Xcalibur software was used to analyse the mass spectra and calculate intensities for generation of calibration curves and $\mathrm{K}_{\mathrm{D}}$ determination from equilibrium microdialysis.

\subsection{Computational Design-Simulating System Behaviour}

The previous microdialysis analysis method presented by Weidemann et al. [12] was based on solving the necessary differential equations followed by simplifications based on the assumption that the protein is in large excess over the small molecule tested. Here we present the derivation of a complete system definition achievable using the symbolic manipulation abilities of Wolfram Mathematica (version 12.1) [17]. Additionally, we included a term to correct for the possible non-complete equilibrium of test compounds. Whilst a strongly and indefinitely non-equilibrating compound (see for reasons above) should be excluded from the assay, the inclusion of this term performs a correction for compounds, which would achieve equilibrium if given enough time. To arrive at the underlying equations governing a microdialysis experiment, we defined three locations in which compound can exist. These are: free in the white chamber (since there is no protein), free in the red chamber (unbound but in the presence of protein) and finally a third virtual compartment-bound to protein in the red chamber. The system can thus be defined by the following equations.

$$
\begin{gathered}
l 0 *(\text { whitevol }+ \text { redvol })==((\text { lwhite } * \text { whitevol })+((\text { fred }+t l) * \text { redvol })), \\
l \text { fred }==p_{c} * \text { lwhite }, \\
t l * \mathrm{~K}_{\mathrm{D}}==t f *(\text { lfred }), \\
\text { lred }==\text { lfred }+t l, \\
p_{t}==\text { lred } / \text { lwhite }
\end{gathered}
$$

where 10 is the ligand concentration representing an equal distribution of ligand across both sides of the partition between red and white volumes at equilibrium, following an amount of ligand being added to the red volume in the absence of the protein. whitevol and redvol are the volumes of the red and white chambers, respectively. lfred and lwhite are the concentrations of free ligand in the red and white chambers, respectively. $t l$ is the concentration of target-ligand complex in the red 
chamber. $t f$ is the concentration of free (unbound to ligand) target protein in the red chamber. $K_{D}$ is the dissociation constant of the protein-ligand (target-ligand) complex. lred is the overall concentration of the compound in the red chamber, including the bound and unbound compound. $p_{t}$ is the ratio between lred and lwhite. $p_{c}$ is the ratio of the compound concentrations in the red and white chambers in the control experiment in the absence of the protein; ideally this should be equal to 1 as both of these concentrations would be 10 .

This system of equations for lred, lwhite, $p_{t}$ and $\mathrm{K}_{\mathrm{D}}$ can be solved using Wolfram Mathematica (version 12.1), allowing it to be fully simulated for experimental planning. Three equations for $K_{D}$ determination can be produced using each of the three experimentally determined variables: lred, lwhite and $p_{t}$. This enables the $\mathrm{K}_{\mathrm{D}}$ to be determined using a single concentration sample from either of the chambers, or by sampling both and determining a $p_{t}$. In addition, the Python [18] code can be used to perform the simulation of custom microdialysis experiments, reproduce the simulation plots within this protocol and explore numerous examples; this is freely available under the open source MIT license at https:/github.com/stevenshave/microdialysis.

The above equations defining the system may be manipulated by Mathematica to remove unknowns and solve for specific member variables. The following code recreates the system:

$$
\begin{aligned}
& \text { model }=t 0==t f+t l, l 0 *(\text { whitevol }+ \text { redvol })= \\
& =\text { lwhite } * \text { whitevol }+ \text { lred } * \text { redvol, lfred }==p c * l \text { white, } t l * \mathrm{~K}_{\mathrm{D}} \\
& ==t f *(\text { lfred }), \text { lred }==\text { lfred }+t l, p t==\text { lred } / \text { lwhite; }
\end{aligned}
$$

Which may then be solved for different member variables with intermediate steps of elimination for unknown variables such as $l f r e d, t l, t f$ and more, depending on the subject of the formula.

$$
\begin{gathered}
\text { Solve }[\text { Simplify }[\text { Eliminate }[\text { model, } t f, t l, l \text { fred, lwhite, } p t]], \text { lred }][[1]] \\
\text { Solve }[\text { Simplify }[\text { Eliminate }[\text { model, } t f, t l, l \text { fred, lred, } p t]], \text { lwhite }][[2]] \\
\text { Solve }[\text { Simplify }[\text { Eliminate }[\text { model, }\{t f, t l, l \text { fred, lwhite, moleslred, moleslwhite, lred }\}]], p t][[2]]
\end{gathered}
$$

Notice that in the above calls to Mathematica's Solve function, we took solutions 1, 2 and finally 2 , as signified by the square brackets at the end of each line, choosing the correct polynomial root produced in an intermediate step determining equations for 1:1 binding. The direct analytical solutions for lred, lwhite and $p_{t}$ are as follows:

$$
\begin{aligned}
& \text { lred }=\frac{1}{2\left(p_{c}{ }^{2} \text { redvol }{ }^{2}+\text { pcvalue } * \text { redvol } * \text { whitevol }\right)} *\left(2 * 10 * p_{c}{ }^{2} * \text { redvol }^{2}\right. \\
& +\mathrm{K}_{\mathrm{D}} * p_{c} * \text { redvol } * \text { whitevol }+10 * p_{c} * \text { redvol } * \text { whitevol }+2 \\
& * 10 * p_{c}^{2} * \text { redvol } * \text { whitevol }+p_{c} * \text { redvol } * t 0 * \text { whitevol } \\
& +\mathrm{K}_{\mathrm{D}} * \text { whitevol }^{2}+10 * p_{c} * \text { whitevol }^{2} \\
& -\sqrt{ }\left(\left(-2 * l 0 * p_{c}{ }^{2} * r e d v o l^{2}-\mathrm{K}_{\mathrm{D}} * p_{c} * \text { redvol } * \text { whitevol }-10\right.\right. \\
& * p_{c} * \text { redvol } * \text { whitevol }-2 * 10 * p_{c}{ }^{2} \text { redvol } * \text { whitevol }-p_{c} \\
& * \text { redvol } * t 0 * \text { whitevol }-\mathrm{K}_{\mathrm{D}} * \text { whitevol }{ }^{2}-10 * p_{c} \\
& \left.* \text { whitevol }^{2}\right)^{2}-4\left(p_{c}{ }^{2} * \text { redvol }{ }^{2}+p_{c} * \text { redvol } * \text { whitevol }\right)\left(10^{2}\right. \\
& * p_{c}^{2} * \text { redvol }{ }^{2}+\mathrm{K}_{\mathrm{D}} * l 0 * p_{c} * \text { redvol } * \text { whitevol }+2 * 10^{2} * p_{c}{ }^{2} \\
& * \text { redvol } * \text { whitevol }+10 * p_{c} * \text { redvol } * t 0 * \text { whitevol }+\mathrm{K}_{\mathrm{D}} * 10 \\
& * p_{c} * \text { whitevol }{ }^{2}+10^{2} * p_{c}^{2} \text { whitevol }{ }^{2}+l 0 * p_{c} * t 0 \\
& \left.\left.* \text { whitevol }^{2}\right)\right) \text { ) }
\end{aligned}
$$




$$
\begin{aligned}
& \text { lwhite }=\frac{1}{2\left(p_{c}^{2} * \text { redvol }+p_{c} * \text { whitevol }\right)} *\left(-\mathrm{K}_{\mathrm{D}} * p_{c} * \text { redvol }+l 0 * p_{c}\right. \\
& * \text { redvol }-p_{c} * \text { redvol } * t 0-\mathrm{K}_{\mathrm{D}} * \text { whitevol }+10 * p_{c} \\
& * \text { whitevol }+\sqrt{ }\left(-4\left(-\mathrm{K}_{\mathrm{D}} * 10 * \text { redvol }-\mathrm{K}_{\mathrm{D}} * 10 * \text { whitevol }\right)\left(p_{c}{ }^{2}\right.\right. \\
& \left.* \text { redvol }+p_{c} * \text { whitevol }\right) \\
& +\left(\mathrm{K}_{\mathrm{D}} * p_{c} * \text { redvol }-10 * p_{c} * \text { redvol }+p_{c} * \text { redvol } * t 0+\mathrm{K}_{\mathrm{D}}\right. \\
& \left.* \text { whitevol }-10 * p_{c} * \text { whitevol) } 2\right) \text { ) }
\end{aligned}
$$

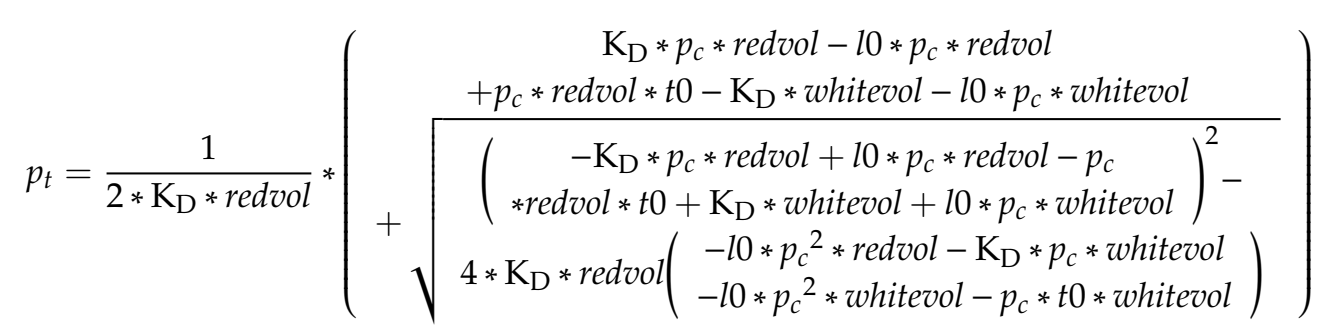

With the simulation of concentrations fully defined, the equations for $\mathrm{K}_{\mathrm{D}}$ determination can be derived, which also contribute to experimental planning aspects. There are three equations for $K_{D}$ determination, either by the measured concentration in the white chamber (lwhite), the red chamber (lred) or a combination of both (expressed as $p_{t}$ ). Whilst simulation and production of concentration plots as shown in Figure 1 would allow determination of $\mathrm{K}_{\mathrm{D}}$ from $y$-axis concentrations and $x$-axis $\mathrm{K}_{\mathrm{D}}$ values, direct analytical solutions for $\mathrm{K}_{\mathrm{D}}$ determination are quicker and more accurate. We may solve the quantitative microdialysis model previously defined for $\mathrm{K}_{\mathrm{D}}$ in terms of lred, lwhite and $p_{t}$, producing three equations for the determination of $\mathrm{K}_{\mathrm{D}}$.

$$
\begin{aligned}
& \text { Solve }\left[\text { Simplify }[\text { Eliminate }[\text { model, } t f, t l, \text { lfred, lwhite, lred }]], \mathrm{K}_{\mathrm{D}}\right] \\
& \text { Solve }\left[\text { Simplify }[\text { Eliminate }[\text { model, } t f, t l, l \text { fred, lwhite, } p t]], \mathrm{K}_{\mathrm{D}}\right] \\
& \text { Solve }\left[\text { Simplify }[\text { Eliminate }[\text { model, } t f, t l, \text { lfred, lred, ptvalue }]], \mathrm{K}_{\mathrm{D}}\right]
\end{aligned}
$$

Producing the following equations for $\mathrm{K}_{\mathrm{D}}$ :

$$
\begin{aligned}
& -10 * p_{c}^{2} r e d v o l+l 0 * p_{c} * p_{t} * \text { redvol } \\
& -p_{c} * p_{t} * \text { redvol } * t 0 \\
& -10 * p_{c}{ }^{2} \text { whitevol }+10 * p_{c} \\
& \mathrm{~K}_{\mathrm{D}} \text { from } p_{t}=\frac{* p_{t} * \text { whitevol }-p_{c} * t 0 * \text { whitevol }}{\left(p_{c}-p_{t}\right)\left(p_{t} * \text { redvol }+ \text { whitevol }\right)} \\
& -l 0^{2} * p_{c}{ }^{2} * r e d v o l^{2}+2 * l 0 * l r e d * p_{c}^{2} * r e d v o l^{2}-\text { lred }^{2} * p_{c}{ }^{2} * r e d v o l^{2} \\
& +10 * \text { lred } * p_{c} * \text { redvol } * \text { whitevol } \\
& - \text { lred }^{2} * p_{c} * \text { redvol } * \text { whitevol }-2 * 10^{2} * p_{c}^{2} * \text { redvol } * \text { whitevol } \\
& +2 * 10 * \text { lred } * p_{c}^{2} * \text { redvol } * \text { whitevol }-10 * p_{c} * \text { redvol } * t 0 * \text { whitevol }
\end{aligned}
$$

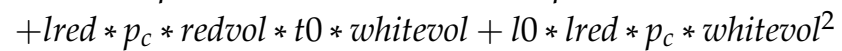

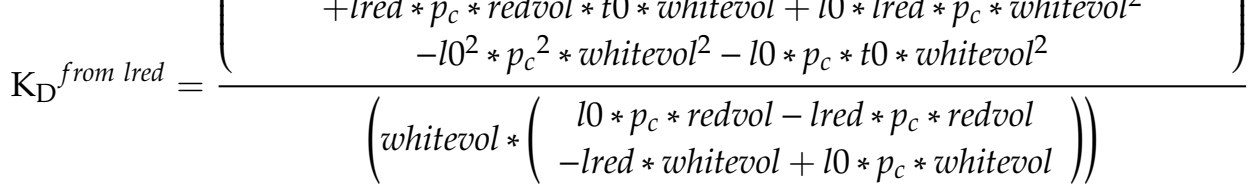

$$
\begin{aligned}
& \text { lwhite } *\left(10 * p_{c} * \text { redvol }- \text { lwhite } * p_{c}{ }^{2} *\right. \text { redvol } \\
& \mathrm{K}_{\mathrm{D}} \text { from lwhite }=-\frac{\left.-p_{c} * \text { redvol } * t 0+10 * p_{c} * \text { whitevol }- \text { lwhite } * p_{c} * \text { whitevol }\right)}{l 0 * \text { redvol }- \text { lwhite } * p_{c} * \text { redvol }+l 0 * \text { whitevol }- \text { lwhite } * \text { whitevol }}
\end{aligned}
$$

These direct analytical solutions for $K_{D}$ allow analysis of experimental data in terms of the compound concentration in either chamber, or the ratio between them. 


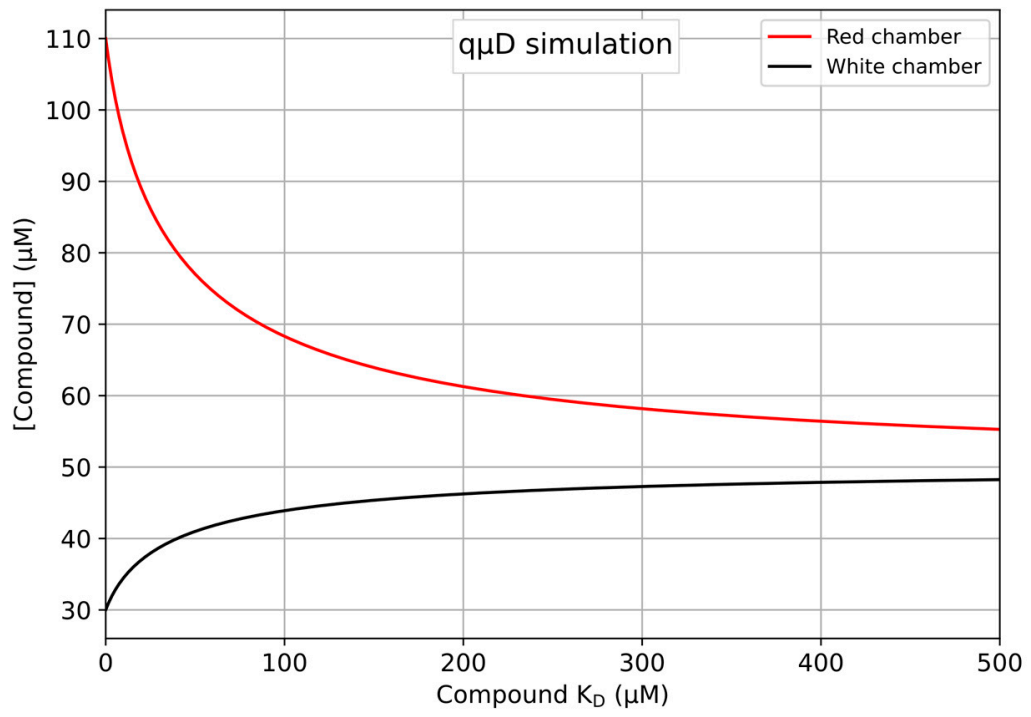

Figure 1. Protein-ligand complex $\mathrm{K}_{\mathrm{D}}$ vs. compound concentration in the red and white chambers. Standard microdialysis system: $100 \mu \mathrm{L}$ red chamber, $300 \mu \mathrm{L}$ white chamber, [ $\mathrm{t} 0]=80 \mu \mathrm{M}$ and [10] $=50$ $\mu \mathrm{M}$. Distinguishing between high $\mathrm{K}_{\mathrm{D}} \mathrm{s}$ becomes difficult as $\mathrm{K}_{\mathrm{D}} \mathrm{s}$ increase as concentrations converge and gradients reduce. Maximum sensitivity and lowest error in $K_{D}$ determination will be present at low $\mathrm{K}_{\mathrm{D}} \mathrm{s}$ (high affinities).

\section{Procedure}

\subsection{Step 1: Obtaining Calibration Curves}

1. Run a RP-HPLC analysis of the small molecules under investigation at $200 \mu \mathrm{M}$ concentration, using the method above to determine the purity and retention time (Rt) of the compound. Additionally, identifying the absorption wavelength that gives the strongest absorption and most stable baseline. We found that this is usually the optical density (OD) at $210 \mathrm{~nm}$ or at $260 \mathrm{~nm}$. Alternatively, for LC-MS, we ran an analysis of the compound at $5 \mu \mathrm{M}$ using the method above with an injection volume of $2 \mu \mathrm{L}$. We determined the Rt and the mass of the protonated molecular ion of the compound $[\mathrm{M}+\mathrm{H}]^{+}$in the ion chromatogram, with $\mathrm{M}$ being the monoisotopic mass of the compound.

2. Create a series of dilutions of the small molecules to be tested in the assay buffer with $5 \%$ DMSO and $0.1 \%$ Pluronic f127 in HPLC insert vials, giving a total volume per concentration of $100 \mu \mathrm{L}$. The concentrations needed to depend on each individual compound. However, we found that a versatile initial choice of concentrations, applicable to most compounds, is 10, 25, 50, 75 and $100 \mu \mathrm{M}$. If LC-MS is to be used instead, a series of dilutions of 50, 100, 500, 1000 and $1500 \mathrm{nM}$ are recommended, in an assay buffer with 5\% DMSO in HPLC insert vials, at a total volume per concentration of $50 \mu \mathrm{L}$. Pluronic f127 is omitted in this instance as detergents ionise very well so can cause problems in mass spectrometry.

3. Run RP-HPLC or LC-MS analyses of the dilution series of compounds, in triplicate, using the method described above. Injection volume $25 \mu \mathrm{L}$ (RP-HPLC) or $2 \mu \mathrm{L}$ (LC-MS) for each concentration.

4. Analyse the data. For RP-HPLC, determine the area under the elution peak at the predetermined $\mathrm{Rt}$ in the particular chromatogram chosen in 3.1.1, and plot peak area versus each of the applied small molecule concentrations in triplicate. The graph should show a straight line if the analysis was performed with compound concentrations in the linear range of the detector. Based on our own analyses, we concluded that the linear range of peak area versus concentration of $>90 \%$ of small molecules tested covered a range between 25 and $200 \mu \mathrm{M}$. Fit the data to a linear $\mathrm{y}=\mathrm{kx}$ $+\mathrm{d}$ equation using a suitable software (Excel, Origin, GraFit, etc.). These preanalysis runs will 
serve as reference information for the expected peak area for each test compound concentration. Alternatively for LC-MS, we obtained the ion chromatogram of the compound and determine the area under the $[\mathrm{M}+\mathrm{H}]^{+}$mass ion peak at its $\mathrm{Rt}$, which was identified in 3.1.1. This ion peak area represents the total ion count; we plotted this against each of the small molecule concentrations in triplicate and followed the same procedure as for HPLC above to obtain a calibration curve. Based on our analyses, the linear range of our LC-MS covered a range between 100 and $1000 \mathrm{nM}$.

\subsection{Step 2: Identification of Appropriate Target Protein Concentration}

The simulation of concentrations present over a range of $K_{D} s$ is performed by using either the accompanying Python code or Microsoft Excel-based workbook; both are available at https://github. com/stevenshave/microdialysis. Alternatively, the equations derived in Section 2.3 (computational design-simulating system behaviour) may be translated into a preferred plotting or simulation environment. Working in a standard unit scale such as micro- or nano- simplifies the input and reduces the risk of numerical instability. Therefore, micromolar and microliter units were chosen as defaults for the input, calculations and output evaluation. Described below are the steps necessary to simulate a 'standard' HPLC-analysis-based microdialysis system using an $80 \mu \mathrm{M}$ protein, a starting ligand concentration of $200 \mu \mathrm{M}$, which equilibrates to $50 \mu \mathrm{M}$ in the absence of protein, and standard red and white volumes of 100 and $300 \mu \mathrm{L}$ respectively. Target-ligand $K_{\mathrm{D}}$ s from (fractionally above) 0 to $500 \mu \mathrm{M}$ were simulated. These simulations served to ensure that unbound compound concentrations achieved at the $\mathrm{K}_{\mathrm{D}} \mathrm{s}$ of interest were accurately readable with the equipment used.

Simulation using the Python code:

1. Open the Python program entitled “02_plot_KDvsConcentrations.py" in a text editor and assign simulation concentrations to the following variables:

a. $\quad 10=50 \rightarrow$ Compound (ligand) concentration achieved at equilibrium in both chambers in the absence of the protein. A starting concentration of $200 \mu \mathrm{M}$ in the red chamber would equilibrate to $50 \mu \mathrm{M}$ in both chambers in a system with standard volumes of 100 and 300 $\mu \mathrm{L}$ for the red and white chambers respectively.

b. $\quad \mathrm{t} 0=80 \rightarrow$ Protein (target) concentration in the red chamber.

c. $\quad$ redvol $=100 \rightarrow$ Volume of the red chamber.

d. $\quad$ whitevol $=300 \rightarrow$ Volume of the white chamber.

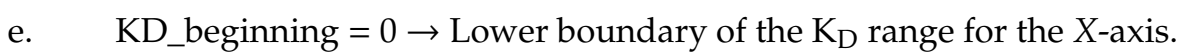

f. $\quad$ KD_end $=500 \rightarrow$ Upper boundary of the $\mathrm{K}_{\mathrm{D}}$ range for the $X$-axis.

g. $\quad \mathrm{pc}=1.0 \rightarrow p_{c}=1$ indicates that the compound equilibrates perfectly in the control quD run.

2. Save and run the newly edited Python program to produce a plot as shown in Figure 1.

3. To perfom single point accurate measurements and read out the data, edit the Python program “01_simulate_qud_concentrations.py" and set 10, t0, red and whitevol as defined in the Python part of Section 3.2, additionally, set kdtl to be the precise value of $K_{D}$ at which to read out the red and white volume concentrations.

Simulation using the Excel workbook:

1. Open the Excel workbook file entitled "quDSimulation_v1.xlsx" in Microsoft Excel, LibreOffice calc or a similar compatible spreadsheet program. Fields that should be edited by users to simulate a system are coloured red, and readout cells are green.

2. Open the sheet entitled 'Simulation' and edit the following fields as shown below to simulate the standard $q \mu \mathrm{D}$ system defined above. The plot produced automatically updates upon eachvariable manipulation.

a. $\quad 10=50 \rightarrow$ Compound (ligand) concentration achieved at equilibrium in both chambers in the absence of the protein. A starting concentration of $200 \mu \mathrm{M}$ in the red chamber would 
equilibrate to $50 \mu \mathrm{M}$ across chambers in a system with standard volumes of 100 and 300 $\mu \mathrm{L}$ for red and white chambers respectively.

b. $\quad \mathrm{t} 0=80 \rightarrow$ Protein (target) concentration in the red chamber.

c. $\quad$ redvol $=100 \rightarrow$ Volume of the red chamber.

d. $\quad$ whitevol $=300 \rightarrow$ Volume of the white chamber.

e. $\quad$ KD_end $=500 \rightarrow$ Upper boundary of the $K_{D}$ range for the $X$-axis.

f. $\quad \mathrm{pc}=1.0 \rightarrow p_{c}=1$ indicates that the compound equilibrates perfectly in the control $q \mu \mathrm{D}$ run.

3. The updated plot is displayed automatically on the sheet, producing the plot shown in Figure 1 .

4. To perfom accurate single-point measurements and read out the data, select the 'KD to conc+pValue' sheet and fill in the same variables as listed in the Excel part of Section 3.2, adding a new value for ' $K D$ ' indicating the $K_{D}$ at which to read out the red and white chamber ligand concentrations.

\subsection{Step 3: Compound-only Dialisability Test}

1. Define the compound concentration to use based on the peak area as a function of the concentration analysis performed in 3.1.4; it should lie in the middle of the linear range determined in 3.1.4. Keep in mind that after full equilibration only $25 \%$ of the starting concentration will be detectable (see $\mathrm{q} \mu \mathrm{D}$ process outline above). For example, if the centre of the linear range found is $50 \mu \mathrm{M}$ for the RP-HPLC analysis, then one needs to use a starting compound concentration of $200 \mu \mathrm{M}$. This is a direct consequence of the volumes present in the RED device.

2. Prepare at least $900 \mu \mathrm{L}$ of the assay buffer. When RP-HPLC is used for analysis, this includes $5 \%$ DMSO and $0.1 \%$ pluronic f127. For LC-MS, Pluronic f127 is omitted.

3. To produce the standard $q \mu \mathrm{D}$ system modelled in Section 3.2, step 2, prepare a $350 \mu \mathrm{L}$ solution of test compounds at $200 \mu \mathrm{M}$ in the assay buffer with $5 \%$ DMSO and $0.1 \%$ pluronic f127. Apply sonication and vortexing to support compound solubilisation, and visually inspect the solution for precipitation. For LC-MS, prepare a $350 \mu \mathrm{L}$ solution of test compounds at $40 \mu \mathrm{M}$ in the assay buffer with $5 \%$ DMSO and use the same methods as above to make sure that the compound is fully in solution.

4. Clean the base plate thoroughly with $20 \%$ ethanol and allow it to dry.

5. Place 3 RED devices into the base plate for each compound to be tested.

6. Add $100 \mu \mathrm{L}$ of compound solution into the red chamber of each RED device and $300 \mu \mathrm{L}$ of assay buffer with $5 \%$ DMSO and $0.1 \%$ pluronic f127 in the white chamber, respectively. For LC-MS: add $100 \mu \mathrm{L}$ of compound solution into the red chamber of each RED device and $300 \mu \mathrm{L}$ of assay buffer with $5 \%$ DMSO.

7. Seal the base plate with a self-adhesive plate seal to inhibit evaporation.

8. Place the base plate onto an orbital shaker at $250 \mathrm{rpm}$ for $6 \mathrm{~h}$ for equilibration. Up to $6 \mathrm{~h}$ is the suggested time by the manufacturers but this needs to be investigated for the compound under study; some compounds may require an overnight equilibration.

9. After $>6 \mathrm{~h}$ equilibration time, harvest $50 \mu \mathrm{L}$ from each chamber of each RED tube and run RP-HPLC using the identical gradient and $25 \mu \mathrm{L}$ injection volume. If fully equilibrated, the compound concentration in either chamber should be $50 \mu \mathrm{M}$. For LC-MS, harvest $10 \mu \mathrm{L}$ from each chamber of the RED tube, and dilute $20 \times$ with $0.1 \%$ formic acid solution. Run the diluted solution using the LC-MS method above, injection volume $2 \mu \mathrm{L}$. If fully equilibrated, the compound concentration in either chamber should be $10 \mu \mathrm{M}$ and after $20 \times$ dilution it should be $500 \mathrm{nM}$.

10. Check that the Rt in the chromatographic elution profile of each test sample has not changed from the condition-setting experiments. Determine the peak area for the compounds in the solutions taken from the red and the white chamber for each HPLC run. Determine $p_{c}$ from the ratio of these two peak areas. A well-equilibrated compound should result in a $p_{c}$ of $0.90-1.10$. Any compound showing larger than $10 \%$ deviation from 1.00 should be considered for exclusion from 
further analysis. For LC-MS, check that the Rt in the ion chromatogram of each sample has not changed from the condition setting experiments. Determine the mass ion peak area for the compounds in the solutions taken from the red and white chamber for each LC-MS run. Perform a data analysis as above using this total ion count.

11. Additionally, if a compound shows full equilibration with $0.90<p_{c}<1.10$, check for compound losses, which could occur for a variety of reasons described in the introduction section. If the area under the chromatographic peaks (or mass ion peak in LC-MS) is within 10\% of the one found during the initial calibration experiment using a $50 \mu \mathrm{M}(500 \mathrm{nM}$ for LC-MS) concentration, it can be assumed that compound losses are negligible.

\subsection{Step 4: Microdialysis Target Protein-Small Molecule Binding assay}

1. Place 6 RED devices (3 RED devices containing compounds only, and 3 containing compounds + target protein) into the cleaned base plate.

2. Prepare $2 \mathrm{~mL}$ of assay buffer with $5 \%$ DMSO and $0.1 \%$ pluronic $f 127$ when HPLC is used for analysis. When LC-MS is used for analysis, prepare $2 \mathrm{~mL}$ of the assay buffer containing $5 \%$ DMSO.

3. Prepare $350 \mu \mathrm{L}$ of $200 \mu \mathrm{M}$ small molecule solution in the assay buffer with 5\% DMSO and $0.1 \%$ pluronic f127, and $350 \mu \mathrm{L}$ of $200 \mu \mathrm{M}$ small molecule plus $80 \mu \mathrm{M}$ protein in the same buffer. For LC-MS, prepare $350 \mu \mathrm{L}$ of $40 \mu \mathrm{M}$ small molecule solution in the assay buffer with $5 \%$ DMSO and $350 \mu \mathrm{L}$ of $40 \mu \mathrm{M}$ small molecule plus $40 \mu \mathrm{M}$ protein in the same buffer.

4. Add $3 \times 100 \mu \mathrm{L}$ of $200 \mu \mathrm{M}$ (or $40 \mu \mathrm{M}$ ) compound solution to 3 red chambers of the RED tubes followed by adding $3 \times 300 \mu \mathrm{L}$ dialysis buffer in the corresponding white chambers.

5. Add $3 \times 100 \mu \mathrm{L}$ of $200 \mu \mathrm{M}+80 \mu \mathrm{M}$ protein (or $40 \mu \mathrm{M}$ compound $+40 \mu \mathrm{M}$ protein) solution into 3 red chambers of the RED tubes and add $3 \times 300 \mu \mathrm{L}$ of dialysis buffer in the corresponding white chambers.

6. Seal plate with the self-adhesive plate seal and place on shaker at $250 \mathrm{rpm}$ for $6 \mathrm{~h}$.

7. After $6 \mathrm{~h}$ or overnight incubation, remove the plate from the shaker and harvest $50 \mu \mathrm{L}$ each from the red and white chamber of the compound-only samples, as well as $50 \mu \mathrm{L}$ from the white chambers of the compound-protein binding samples; and add them to insert vials for the HPLC analysis. For the LC-MS analysis, take out $10 \mu \mathrm{L}$ from each red and white chamber of the compound alone samples as well as from the compound and protein samples.

8. Run these samples on RP-HPLC under the same conditions as above, with an injection volume of $25 \mu \mathrm{L}$, or for LC-MS, dilute the $10 \mu \mathrm{L}$ aliquot $20 \times$ with $0.1 \%$ FA solution and run the LC-MS analysis, injection volume $2 \mu \mathrm{L}$.

9. From the resulting chromatograms, obtain peak areas at known Rts for the samples for RP-HPLC. For LC-MS, obtain mass ion peak areas of the compounds at known Rts.

For compound-only experiments:

10. Determine $p_{c}=$ compound peak area from red chamber/white chamber or determine $p_{c}=$ mass ion peak area from red chamber/white chamber.

11. This should resemble the data achieved from the compound equilibration test experiments and should therefore be close to 1.0 .

12. For determination of the total amount of compounds in the quD. Convert the compound peak area or mass ion peak area obtained in Section 3.3. 11 into compound concentration by using the calibration curve in Section 3.1. 4. The amount of compound in the chambers can be obtained by multiplication of the converted compound concentration in the red chamber by $100 \mu \mathrm{L}$ plus the converted compound concentration of the white chamber multiplied by $300 \mu \mathrm{L}$. This should yield close to the amount of introduced compound at the start of the dialysis $(200 \mu \mathrm{M} \times 100 \mu \mathrm{L}=$ $20 \times 10^{-9} \mathrm{~mol}$ for HPLC and $40 \mu \mathrm{M} \times 100 \mu \mathrm{L}=4 \times 10^{-9} \mathrm{~mol}$ for LC-MS) indicating no losses due to adhesion, precipitation or aggregation effects. Note that for LC-MS the solutions were 
diluted $20 \times$ before analysis. Therefore, one needs to multiply by a factor of 20 to obtain the real concentrations in the RED tube.

For compound-target protein binding experiments:

13. From the HPLC chromatograms of the white chambers, determine the peak area and convert to compound concentration by using the calibration curve above.

14. Determine the amount of compound in the white chamber by multiplication of the determined compound concentration with the volume of the white chamber, $300 \mu \mathrm{L}$.

15. From Section 3.3. 13 the total amount of compound is known. Therefore, the amount of compound in the red chamber is the difference between the total amount of compound and the amount of compound in the white chamber.

16. Calculated the compound concentration in the red chamber by dividing the amount of compound in the red chamber by $100 \mu \mathrm{L}$.

17. Determine $p_{t}$ by dividing the compound concentration of the red chamber by that of the white chamber. For LC-MS, determine $p_{t}$ by dividing the compound mass ion peak area of the red chamber by that of the white chamber obtained in Section 3.3. 9.

\subsection{Step 5: Use the Software to Determine the Dissociation Constant $\left(K_{D}\right)$ of the Compound to the Target} Protein by Using the Red/White Chamber Concentrations or $p_{t}$ Values

Determination of the dissociation constants $\left(\mathrm{K}_{\mathrm{D}} \mathrm{s}\right)$ can be performed by either using the accompanying Python code or Microsoft Excel-based workbook, both available at https:/github. com/stevenshave/microdialysis. Below we performed the steps necessary to determine a $\mathrm{K}_{\mathrm{D}}$ for a 'standard' HPLC-analysis-based microdialysis system using $80 \mu \mathrm{M}$ protein, a starting ligand concentration of $200 \mu \mathrm{M}$, equilibrating to $50 \mu \mathrm{M}$ in the absence of protein and standard red and white volumes of 100 and $300 \mu \mathrm{L}$ respectively. There were three equations for $K_{D}$ determination, either by the measured concentration in the white chamber (lwhite), the red chamber (lred), or a combination of both, as expressed as $p_{t}$.

$\mathrm{K}_{\mathrm{D}}$ determination using the Python code on single point measurement data:

1. To determine a $K_{D}$ based on only a single point measurement of the white chamber concentration, open the Python file entitled '03_deriveKD_from_lwhite.py' in a text or code editor and assign the following variables, taking care to input the correct value for lwhite as the measured concentration of ligand in the white chamber and run the program to read out the $\mathrm{K}_{\mathrm{D}}$ :

a. $\quad 10=50$, Compound (ligand) concentration achieved at equilibrium in both chambers in the absence of the protein. A starting concentration of $100 \mu \mathrm{M}$ in the red chamber would equilibrate to $25 \mu \mathrm{M}$ across chambers in a system with standard volumes of 100 and $300 \mu \mathrm{L}$ for red and white chambers respectively.

b. $\mathrm{t} 0=80$, Protein (target) concentration present in the red chamber.

c. redvol $=100$, Volume of the red chamber.

d. $\quad$ whitevol $=300$, Volume of the white chamber.

e. lwhite, The ligand concentration as measured in the white chamber.

f. $\quad \mathrm{pc}, p_{c}=1$ indicates that the compound equilibrates perfectly in the control $q \mu \mathrm{D}$ run.

2. To determine a $K_{D}$ based on only a single point measurement of the red chamber concentration, open the Python file entitled '03_deriveKD_from_lred.py' in a text or code editor and assign the following variables, taking care to input the correct value for lred as the measured concentration of ligand in the red chamber and run the program to read out the $\mathrm{K}_{\mathrm{D}}$ :

a. $10=50$, Compound (ligand) concentration achieved at equilibrium in both chambers in the absence of the protein. A starting concentration of $100 \mu \mathrm{M}$ in the red chamber would 
equilibrate to $25 \mu \mathrm{M}$ across chambers in a system with standard volumes of 100 and $300 \mu \mathrm{L}$ for red and white chambers respectively.

b. $t 0=80$, Protein (target) concentration present in the red chamber.

c. $\quad$ redvol $=100$, Volume of the red chamber.

d. $\quad$ whitevol $=300$, Volume of the white chamber.

e. lred, The ligand concentration as measured in the red chamber.

f. $\quad \mathrm{pc}, p_{c}=1$ indicates that the compound equilibrates perfectly in the control $q \mu \mathrm{D}$ run.

3. To determine a $K_{D}$ based on a $p_{t}$ derived from single point measurements open the Python file entitled '03_deriveKD_from_pt.py' in a text or code editor and assign the following variables, taking care to input the correct value for $\mathrm{pt}$ as the ratio of compound concentration the the red versus white chambers and run the program to read out the $\mathrm{K}_{\mathrm{D}}$ :

a. $\quad 10=50$, Compound (ligand) concentration achieved after equilibrium in both chambers in the absence of the protein. A starting concentration of $100 \mu \mathrm{M}$ in the red chamber would equilibrate to $25 \mu \mathrm{M}$ across chambers in a system with standard volumes of 100 and $300 \mu \mathrm{L}$ for red and white chambers respectively.

b. $\quad t 0=80$, Protein (target) concentration present in the red chamber.

c. $\quad$ redvol $=100$, Volume of the red chamber.

d. $\quad$ whitevol $=300$, Volume of the white chamber.

e. $\mathrm{pt}$, The $p_{t}$ value, which is the concentration of ligand in the red chamber divided by the concentration of ligand in the white chamber.

f. $\quad \mathrm{pc}, p_{c}=1$ indicates that the compound equilibrates perfectly in the control $q \mu \mathrm{D}$ run.

$\mathrm{K}_{\mathrm{D}}$ determination using the Python code on multiple replicates of measurement data:

1. To determine a $K_{D}$ based on multiple replicate measurements of compound concentration in the white chamber, open '04_deriveKD_from_multiple_lwhite.py' in a text or code editor. Comments in the file provide instructions in line with the following:

a. Assign the experimental variables as descibed in Section 3.5. 1 above, setting 10, t0, redvol and whitevol.

b. If 3 measurements of compound concentration lwhite have been made, calculate the mean and standard deviation of these variables using numpy (val1, val2 and val3 bellow are measurement replicates):

i. $\quad$ lwhite_mean $=$ np.mean $([v a l 1$, val2, val3] $)$;

ii. $\quad$ lwhite_std $=$ np.std([val1, val2, val3]).

c. Assign lwhite to be a newly constructed Python uncertainties package ufloat object.

i. $\quad$ lwhite $=$ ufloat $\left(1 w h i t e \_m e a n\right.$, lwhite_std $)$.

d. If multiple $p_{c}$ values have been derived, the pc variable may be assigned to a ufloat object constructed as:

i. $\quad$ pc_mean $=$ np.mean $([\operatorname{val} 1$, val2, val3] $)$;

ii. $\quad$ pc_std $=\operatorname{np} . s t d([\operatorname{val1}$, val2, val3]);

iii. $\quad \mathrm{pc}=$ ufloat(pc_mean, $\mathrm{pc} \_$std) .

e. Alternatively, a single $p_{c}$ value may be assigned as in 3.5.

2. To determine a $\mathrm{K}_{\mathrm{D}}$ based on multiple replicate measurements of compound concentration in the red chamber, open '04_deriveKD_from_multiple_lred.py' in a text or code editor. Comments in the file provide instructions in line with the following: 
a. Assign the experimental variables as descibed in Section 3.5. 1 above, setting 10, t0, redvol and whitevol.

b. If 3 measurements of compound concentration lred have been made, calculate the mean and standard deviation of these variables using numpy (val1, val2 and val3 bellow are measurement replicates):

i. $\quad$ lred_mean $=$ np.mean $([v a l 1$, val2, val3] $)$;

ii. $\quad$ lred_std $=\operatorname{np} . \operatorname{std}([\operatorname{val1}$, val2, val3] $)$.

c. Assign lwhite to be a newly constructed Python uncertainties package ufloat object.

i. $\quad$ lred $=$ ufloat(lred_mean, lred_std).

d. If multiple $p_{c}$ values have been derived, the pc variable may be assigned to a ufloat object constructed as:

i. $\quad$ pc_mean $=$ np.mean $([\operatorname{val1}$, val2, val3] $)$;

ii. $\quad$ pc_std $=\operatorname{np} . \operatorname{std}([\mathrm{val1}, \mathrm{val2}, \mathrm{val3}])$;

iii. $\quad p c=$ ufloat(pc_mean, pc_std).

e. Alternatively, a single $p_{c}$ value may be assigned as in Section 3.5. 1.

3. To determine a $\mathrm{K}_{\mathrm{D}}$ based on multiple replicate measurements of $p_{t}$, open '04_deriveKD_from_multiple_pt.py' in a text or code editor. Comments in the file provide instructions in line with the following:

a. Assign the experimental variables as descibed in Section 3.5. 1 above, setting 10, t0, redvol, and whitevol.

b. If 3 measurements of $p_{t}$ have been made, calculate the mean and standard deviation of these variables using numpy (val1, val2 and val3 bellow are measurement replicates):

i. $\quad$ pt_mean $=$ np.mean $([\mathrm{val1}, \mathrm{val2}, \mathrm{val3}])$;

ii. $\quad$ pt_std $=\operatorname{np} . \operatorname{std}([\mathrm{val1}, \mathrm{val2}, \mathrm{val3}])$.

c. Assign lwhite to be a newly constructed Python uncertainties package ufloat object.

i. $\quad$ pt $=$ ufloat(pt_mean, pt_std).

d. If multiple $p_{c}$ values have been derived, the pc variable may be assigned to a ufloat object constructed as:

i. $\quad$ pc_mean $=$ np.mean $([\operatorname{val1}$, val2, val3] $)$;

ii. $\quad$ pc_std $=\operatorname{np} . \operatorname{std}([\operatorname{val1}$, val2, val3] $)$;

iii. $\quad$ pc $=$ ufloat $\left(p c \_m e a n, p c \_s t d\right)$.

e. $\quad$ Alternatively, a single $p_{c}$ value may be assigned as in Section 3.5. 1 .

$\mathrm{K}_{\mathrm{D}}$ determination using the Excel workbook code:

1. To determine a $K_{D}$ based on only the white chamber concentration, open the Excel file entitled 'quDSimulation_v1.xlsx' in Microsoft Excel, LibreOffice calc, or similar compatible spreadsheet program. Go to the tab named 'lwhite to KD' and assign the following variables, taking care to input the correct value for lwhite as the measured concentration of ligand in the white chamber. The readout of $K_{D}$ will update with every change of input variable:

a. $\quad 10=50 \rightarrow$ Compound (ligand) concentration achieved at equilibrium in both chambers in the absence of the protein. A starting concentration of $100 \mu \mathrm{M}$ in the red chamber would equilibrate to $25 \mu \mathrm{M}$ across chambers in a system with standard volumes of 100 and $300 \mu \mathrm{L}$ for red and white chambers respectively. 
b. $\mathrm{t} 0=80 \rightarrow$ Protein (target) concentration present in the red chamber.

c. $\quad$ redvol $=100 \rightarrow$ Volume of the red chamber.

d. $\quad$ whitevol $=300 \rightarrow$ Volume of the white chamber.

e. $\quad$ lwhite $\rightarrow$ The ligand concentration as measured in the white chamber.

f. $\quad p c \rightarrow p_{c}=1$ indicates that the compound equilibrates perfectly in the control $q \mu D$ run.

2. To determine a $K_{D}$ based on only the red chamber concentration, open the Excel file entitled 'quDSimulation_v1.xlsx' in Microsoft Excel, LibreOffice calc, or similar compatible spreadsheet program. Go to the tab named 'lred to $\mathrm{KD}^{\prime}$ ' and assign the following variables, taking care to input the correct value for LRED as the measured concentration of ligand in the red chamber. The readout of KD will update with every change of input variable:

a. $\quad 10=50 \rightarrow$ Compound (ligand) concentration achieved at equilibrium in both chambers in the absence of the protein. A starting concentration of $100 \mu \mathrm{M}$ in the red chamber would equilibrate to $25 \mu \mathrm{M}$ across chambers in a system with standard volumes of 100 and $300 \mu \mathrm{L}$ for red and white chambers respectively.

b. $\quad \mathrm{t} 0=80 \rightarrow$ Protein (target) concentration present in the red chamber.

c. $\quad$ redvol $=100 \rightarrow$ Volume of the red chamber.

d. $\quad$ whitevol $=300 \rightarrow$ Volume of the white chamber.

e. $\quad$ lred $\rightarrow$ The ligand concentration as measured in the red chamber.

f. $\quad p c \rightarrow p_{c}=1$ indicates that the compound equilibrates perfectly in the control $q \mu D$ run.

3. To determine a $K_{\mathrm{D}}$ based on $p_{t}$, open the Excel file entitled 'quDSimulation_v1.xlsx' in Microsoft Excel, LibreOffice calc or a similar compatible spreadsheet program. Go to the tab named 'pt to $\mathrm{KD}^{\prime}$ and assign the following variables, taking care to input the correct value for PT_VALUE as the measured and calculated $p_{t}$ value. The readout of KD will update with every change of input variable:

a. $\quad 10=50 \rightarrow$ Compound (ligand) concentration achieved at equilibrium in both chambers in the absence of the protein. A starting concentration of $100 \mu \mathrm{M}$ in the red chamber would equilibrate to $25 \mu \mathrm{M}$ across chambers in a system with standard volumes of 100 and $300 \mu \mathrm{L}$ for red and white chambers respectively.

b. $\quad \mathrm{t} 0=80 \rightarrow$ Protein (target) concentration present in the red chamber.

c. $\quad$ redvol $=100 \rightarrow$ Volume of the red chamber.

d. $\quad$ whitevol $=300 \rightarrow$ Volume of the white chamber.

e. $\quad \mathrm{pt} \rightarrow$ The $p_{t}$ value, which is concentration of the ligand in the red chamber divided by the concentration of the ligand in the white chamber.

f. $\quad p c \rightarrow p_{c}=1$ indicates that the compound equilibrates perfectly in the control $q \mu D$ run.

\section{Expected Results}

\subsection{Simulation of the $q \mu D$ Experiment as a Function of the Concentrations and $K_{D} S$}

With equations established to simulate the concentration of compound present in each chamber, the expected red and white chamber concentrations can be evaluated in relation to the sensitivity of the chosen instruments. Since $K_{D}$ determination is achieved by the accurate comparison of compound concentrations in the two chambers, or by inferring either one from the other in the case of only using one concentration, it is important that the experimental setup is such that a sensitive quantitative and accurate concentration determination is possible over the range of conditions of most interest. This may direct the experimental design to search for high or low affinity hits in screening campaigns and directly influences assay sensitivity, threshold values and $Z^{\prime}$ values as an assessment of assay quality. 
Figure 1 demonstrates how compound concentrations in each of the microdialysis chambers converged as the $\mathrm{K}_{\mathrm{D}}$ increased. The simulation was performed as step 2 of the protocol and is necessary to understand the range of $\mathrm{K}_{\mathrm{D}}$ s resolvable as a function of concentrations and the accuracy of the instruments used to measure them. The simulation shows a typical setup for readout via HPLC, in which $80 \mu \mathrm{M}$ of target protein was placed into the red chamber, along with an amount of ligand, which in the absence of the protein would equilibrate to $50 \mu \mathrm{M}$. Large separation of concentrations at low $\mathrm{K}_{\mathrm{D}} \mathrm{S}$ (high affinity) allowed for a confident and accurate assignment of $\mathrm{K}_{\mathrm{D}}$ from concentrations. At the upper end of the simulated $K_{D}$ range between 400 and $500 \mu \mathrm{M}$, the concentration differed minimally, making a direct assignment of $K_{D}$ within this range less accurate. This difficulty in confidently assigning high $\mathrm{K}_{\mathrm{D}} \mathrm{s}$ is further demonstrated in Scheme 1, Step 5, whereby $p_{t}$ is calculated and shown to be converging to what would be $p_{c}$.

Figure 2 further demonstrated the difficulty in using $p_{t}$ to assign high $\mathrm{K}_{\mathrm{D}} \mathrm{s}$, with the $\mathrm{K}_{\mathrm{D}} \mathrm{s}$ of 300 and $500 \mu \mathrm{M}$ producing ptvalues of 1.247 and 1.153 respectively, lred values of 29.366 and 27.760 respectively and lwhite values of 23.545 and $24.081 \mu \mathrm{M}$, respectively.

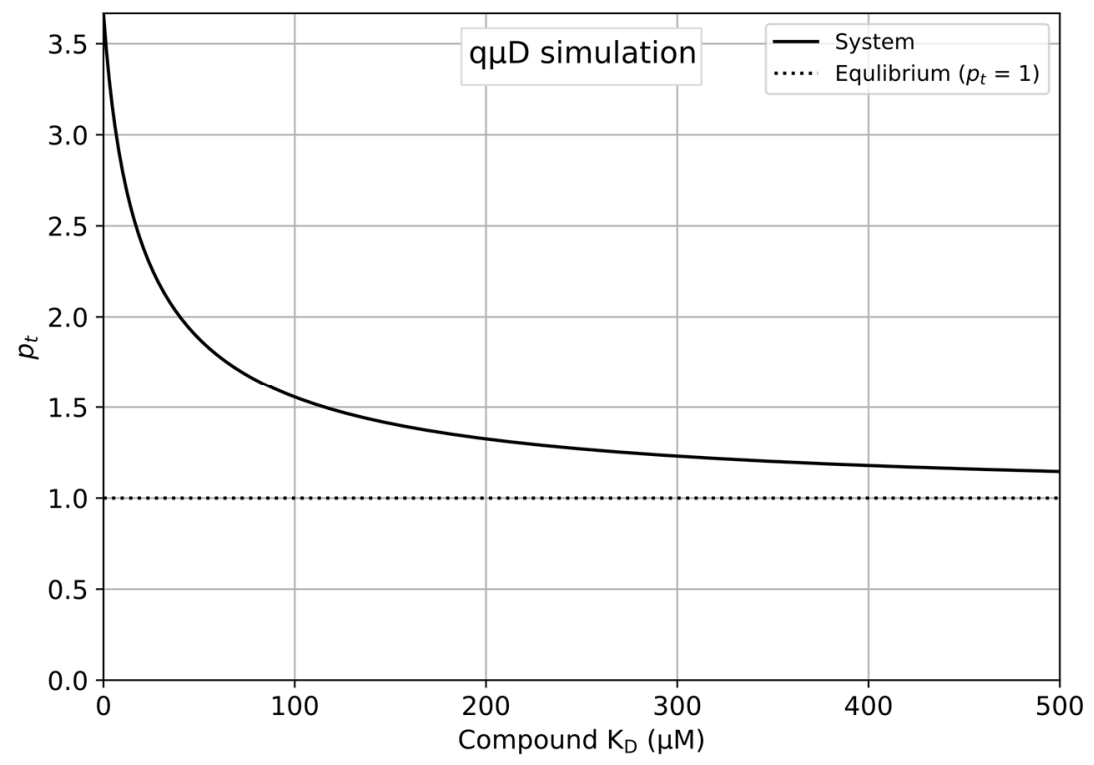

Figure 2. The protein-ligand complex $\mathrm{K}_{\mathrm{D}}$ vs. the $p_{t}$. standard microdialysis system, $100 \mu \mathrm{L}$ red chamber, $300 \mu \mathrm{L}$ white chamber, [t0] $=80 \mu \mathrm{M}$ and [10] $=50 \mu \mathrm{M}$. At higher concentrations, the ratio of the compound within each chamber reaches equilibrium as the $p_{t}$ value becomes asymptotic to $p_{t}=1$. Very precise concentration measurements would be needed to accurately determine high $\mathrm{K}_{\mathrm{D}} \mathrm{s}$ or identify them as hit compounds.

\subsection{Exemplaric Considerations on the Accuracy of $K_{D}$ Determinations}

Differentiating the three equations for $\mathrm{K}_{\mathrm{D}}$ with respect to lred, lwhite and $p_{t}$ allows investigation of the rate of change of $K_{D}$ as a function of changing concentrations, further illustrating the importance of accurate concentration determination. Using the standard microdialysis system simulated in Figures 1 and 2, where $p 0$ was $80 \mu \mathrm{M}, 10$ was $50 \mu \mathrm{M}$ and the standard volumes of 100 and $300 \mu \mathrm{L}$ for the red and white chambers, differentiation with respect to lred, lwhite and $p_{t}$ shows gradients along with concentrations and $p_{t}$ values present at intervals between 1 and $500 \mu \mathrm{M}$. From these values given in Table 1, we clearly see the instantaneous gradient present at red and white concentrations was smaller at $K_{D}$ levels of 1 vs. $500 \mu \mathrm{M} K_{D}$ values. A unit change in red or white concentration invoking almost a 200 -fold instantaneous change in $K_{D}$ at 500 vs. $1 \mu \mathrm{M} K_{D}$. As the change in the $K_{D}$ value as calculated by $p_{t}$ was so large, it was suggested that only one singular concentration be used for $\mathrm{K}_{\mathrm{D}}$ determination. Ideally this would be the red chamber, which had the smallest gradient with respect to the compound concentration present across all $\mathrm{K}_{\mathrm{D}}$ values. However, with protein present, steps for compound 
concentration determination were further extended with the removal of the protein from samples. A general rule is therefore suggested whereby the white chamber compound concentration (lwhite) was used for $\mathrm{K}_{\mathrm{D}}$ determination. The relationship between lred and lwhite for perfectly equilibrating compounds $\left(p_{c}=1.00\right)$ shows identical fold-changes in gradient across $\mathrm{K}_{\mathrm{D}}$ s compared to a baseline gradient at a $K_{D}$ of $1 \mu \mathrm{M}$. We concluded that the $q \mu \mathrm{D}$ method was best suited for determination of $K_{D} S$ less than $200 \mu \mathrm{M}$.

Table 1. Values for lred, lwhite and $p_{t}$, and first derivatives thereof over a range of $\mathrm{K}_{\mathrm{D}} \mathrm{s}$ for the standard system where $p 0$ is $80 \mu \mathrm{M}, 10$ is $50 \mu \mathrm{M}$ and the red and white chambers have standard volumes of 100 and $300 \mu \mathrm{L}$, respectively. A unit change in lred concentration at $55.3 \mu \mathrm{M}\left(\mathrm{K}_{\mathrm{D}}\right.$ of $\left.500 \mu \mathrm{M}\right)$ results in a 193-fold change in $K_{D}$ readout compared to the $108 \mu \mathrm{M}$ concentration $\left(K_{D} 1 \mu \mathrm{M}\right.$ level). Similar changes are seen in the white chamber.

\begin{tabular}{|c|c|c|c|c|c|c|}
\hline \multirow[b]{2}{*}{$K_{D}$} & \multicolumn{2}{|c|}{ lred } & \multicolumn{2}{|c|}{ lwhite } & \multicolumn{2}{|r|}{$p_{t}$} \\
\hline & $\begin{array}{c}\text { Concentration } \\
(\mu \mathrm{M})\end{array}$ & $\begin{array}{c}\partial \mathrm{K}_{\mathrm{D}} / \partial \text { lred } \\
\text { (Fold Change from } \\
1 \mu \mathrm{M})\end{array}$ & $\begin{array}{l}\text { Concentration } \\
\qquad(\mu \mathrm{M})\end{array}$ & $\begin{array}{c}\partial 1 \mathrm{~K}_{\mathrm{D}} / \partial \text { lwhite } \\
\text { (Fold Change from } \\
1 \mu \mathrm{M})\end{array}$ & Value & $\begin{array}{c}\partial \mathrm{K}_{\mathrm{D}} / \partial p_{t} \\
\text { (Fold Change from } \\
1 \mu \mathrm{M})\end{array}$ \\
\hline 1 & 108.1032 & $-0.5553(1)$ & 30.6323 & $1.6659(1)$ & 3.5291 & $-7.8158(1)$ \\
\hline 100 & 68.304 & $-8.6209(16)$ & 43.8987 & $25.8628(16)$ & 1.5559 & $-249.2(32)$ \\
\hline 200 & 61.2679 & $-23.2953(42)$ & 46.244 & 69.8859 (42) & 1.3249 & $-747.259(96)$ \\
\hline 300 & 58.1682 & $-44.6309(80)$ & 47.2773 & $133.8926(80)$ & 1.2304 & -1496.34 (192) \\
\hline 400 & 56.4121 & $-72.6318(131)$ & 47.8626 & 217.8954 (131) & 1.1786 & $-2495.81(319)$ \\
\hline 500 & 55.2795 & $-107.299(193)$ & 48.2402 & 321.8969 (193) & 1.1459 & $-3745.46(479)$ \\
\hline
\end{tabular}

Figure 3 illustrates the possible $K_{\mathrm{D}}$ range calculated using a detection method and handling technique, which introduced a $\pm 5 \%$ (measured at the $2 \sigma$ confidence level) error. The valid $2 \sigma K_{D}$ ranges for the standard HPLC example system used above, with lwhite concentrations of 35, 40 and 45 $\mu \mathrm{M}$ were: 8.9-14.9 $\mu \mathrm{M}, 31.9-50.1 \mu \mathrm{M}$ and 99.4-191.9 $\mu \mathrm{M}$, respectively.

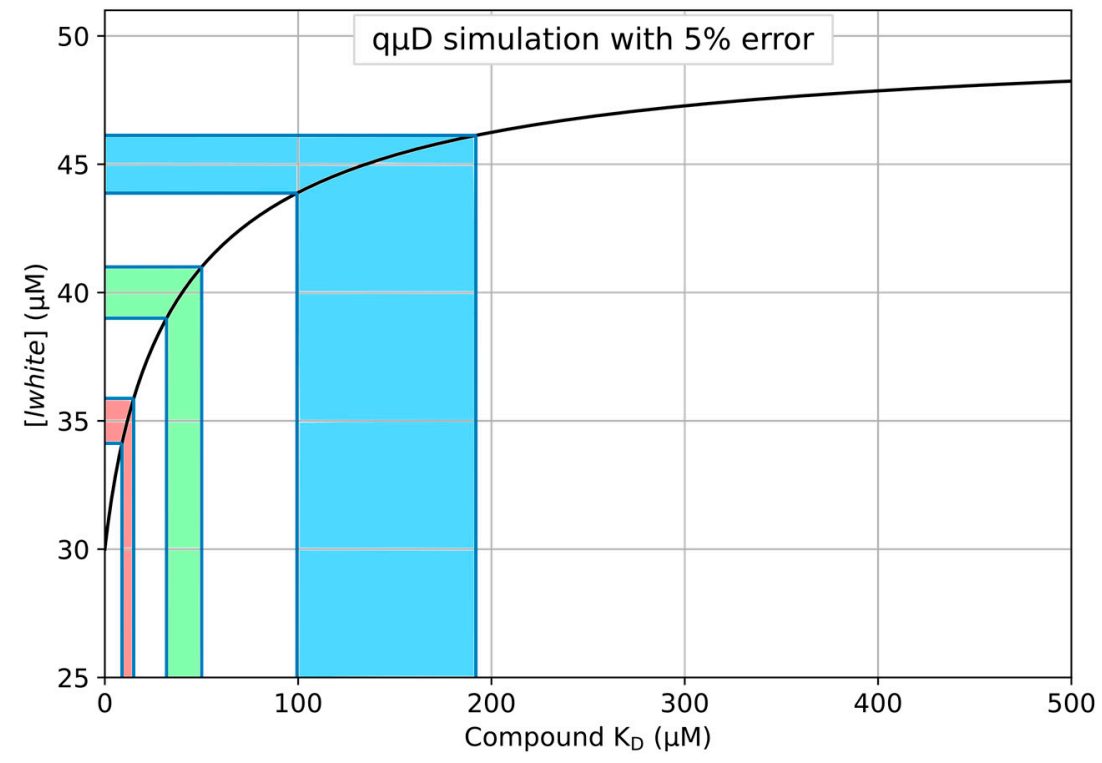

Figure 3. $K_{D}$ determination using a $5 \%$ concentration error. Standard microdialysis system, $100 \mu \mathrm{L}$ red chamber, $300 \mu \mathrm{L}$ white chamber, [t0] $=80 \mu \mathrm{M}$ and [10] = $50 \mu \mathrm{M}$. lwhite compound concentrations of 35 (red), 40 (green) and $45 \mu \mathrm{M}$ (blue) give rise to $\mathrm{K}_{\mathrm{D}}$ ranges of 8.9-14.9 $\mu \mathrm{M}, 31.9-50.1 \mu \mathrm{M}$ and 99.4-191.9 $\mu \mathrm{M}$ respectively.

An accurate determination of $\mathrm{K}_{\mathrm{D}} \mathrm{s}$ will always depend on the accuracy of the concentration determination, which is linked to instrument sensitivity and handling. The impact of these errors may be minimised through proper planning and simulation, knowing the $\mathrm{K}_{\mathrm{D}}$ ranges of most interest and 
setting rejection criteria for hit $\mathrm{K}_{\mathrm{D}} \mathrm{s}$. The mathematical treatment of the microdialysis system as defined in this protocol also increases the accuracy of the technique compared to the previously published simplified equations, which rely on the assumption that the amount of free protein is in great excess of the bound protein. Our protocol does not include such assumptions and represents a correct treatment of the system.

System simulation and reproduction of all plots may be achieved using the supplied Python code or the included Microsoft Excel workbook at https://github.com/stevenshave/microdialysis.

\subsection{Experimental Results}

In the following we summarized the outcome of two $q \mu \mathrm{D}$ experiments, which were performed with primary hits from a plate-based size-exclusion chromatography screen [10] against two validated human cancer targets and one antibacterial target. This method allowed the identification of low affinity binders in the low $\mathrm{mM} \mathrm{K}_{\mathrm{D}}$ range. However, quantification was only relative and exact affinity determination of the target was cumbersome. Additionally, compounds that aggregate in solution at the assay concentrations can result in false positives, since they are spun through a size exclusion membrane as fast as if they were bound to the target protein. Therefore, $q \mu \mathrm{D}$ was applied to check the aggregation and surface binding properties of the primary hit compounds and if suitable, their target binding $\mathrm{K}_{\mathrm{D}}$ s. For compounds $1-3$ the RP-HPLC analysis was applied, for compound 4 the analysis was performed using an LC-MS read out (Table 2).

Table 2. Summary of results obtained with the $q \mu \mathrm{D}$ protocol on compounds, which showed low dialisability (1), nonspecific binding (2), strong specific and weak nonspecific binding (3) and a specific binder, which affinity to the target was compared to the affinity determined by NMR.

\begin{tabular}{ccccccc}
\hline Compound \# & {$[\mathbf{1 0}](\mu \mathbf{M})$} & {$[\mathbf{t 0}](\mu \mathbf{M})$} & $p_{c}$ & $p_{t}$ & $p_{s}$ (BSA) & Comment \\
\hline 1 & 25 & 80 & $1.41 \pm 0.02$ & NA & NA & Non-equilibrating compound. \\
\hline 2 & 25 & 80 & $1.02 \pm 0.01$ & $1.15 \pm 0.03$ & $1.29 \pm 0.05$ & $\begin{array}{c}\text { Equilibrating compound, but it binds } \\
\text { BSA stronger than to the target } \\
\text { protein; } 603 \pm 154 \mu \mathrm{M} \text { vs. } 278 \pm 57 \\
\mu \mathrm{M}-\text { assuming } 1: 1 \text { binding. }\end{array}$ \\
\hline 3 & 25 & 80 & $1.02 \pm 0.01$ & $1.21 \pm 0.01$ & $1.11 \pm 0.01$ & $\begin{array}{c}\text { Equilibrating compound, } \mathrm{K}_{\mathrm{D}}=405 \pm \\
35 \mathrm{M} \text { to target protein, and } 882 \pm 57 \\
\mu \mathrm{M} \text { to BSA-assuming } 1: 1 \text { binding. }\end{array}$ \\
\hline 4 & 10 & 40 & $0.99 \pm 0.03$ & $1.21 \pm 0.04$ & NA & $\begin{array}{c}\text { Well-equilibrating compound, } \mathrm{K}_{\mathrm{D}}= \\
171 \pm 34 \mu \mathrm{M} .\end{array}$ \\
\hline
\end{tabular}

After establishing the calibration curve via RP-HPLC and using the simulation to obtain a suitable protein concentration $(\mathrm{t} 0=80 \mu \mathrm{M})$, the equilibration of compounds in the absence of the protein was performed to obtain a $p_{c}$ value from a $100 \mu \mathrm{M}$ starting compound concentration $(10=25 \mu \mathrm{M})$. After a dialysis time of $6 \mathrm{~h}$ and an overnight incubation $(16 \mathrm{~h})$, the resulting $p_{c}$ value for compound 1 based on a decorated phenylpiperazine scaffold was significantly higher than the set threshold of 1.10. This compound, therefore, never reached equilibrium. Hence, it could not be used for $\mathrm{K}_{\mathrm{D}}$ determination and was excluded from the hit to lead processes.

In contrast, compound 2 based on a pyridin-2-one scaffold equilibrates well with a $p_{c}$ of 1.02 , reached within $6 \mathrm{~h}$ from a starting compound concentration of $100 \mu \mathrm{M}$ and protein concentration of 80 $\mu \mathrm{M}$ in the red chamber $(10=25 \mu \mathrm{M})$. Unfortunately, when the experiment was performed with its target protein and BSA control, $p_{t}$ was less than $p_{s}$ suggesting that the compound binds to BSA with higher affinity than to its target protein with $\mathrm{K}_{\mathrm{D}}$ s of $278 \pm 57 \mu \mathrm{M}$ and $603 \pm 154 \mu \mathrm{M}$ respectively. Compound 3 based on a pyridazine scaffold also equilibrated well with $p_{c}=1.02$. In the presence of $80 \mu \mathrm{M}$ of its target protein and a starting compound concentration of $100 \mu \mathrm{M}(10=25 \mu \mathrm{M})$, q $\mu \mathrm{D}$ yielded a $p_{t}=1.21$. The BSA binding test resulted in $p_{s}=1.11$, suggesting that although this compound demonstrated some low affinity binding with a calculated $\mathrm{K}_{\mathrm{D}}$ of $882 \pm 57 \mu \mathrm{M}$ to BSA, it possessed a higher affinity to its target protein with a $\mathrm{K}_{\mathrm{D}}$ of $405 \pm 35 \mu \mathrm{M}$. The binding of compound 4 also containing a decorated 
phenylpiperazine scaffold had been validated previously using NMR. Therefore, further specificity controls against proteins such as BSA were not carried out at this stage. As seen in Table 2, the $p_{c}$ value was 0.99 , when incubated with $40 \mu \mathrm{M}$ protein and at a starting compound concentration of $40 \mu \mathrm{M}$ $(10=10 \mu \mathrm{M})$, indicating that this compound was fully equilibrated after $16 \mathrm{~h}$ incubation. The $p_{t}$ value in the presence of its target protein was determined to be 1.21, which corresponded to a dissociation constant $\left(K_{D}\right)$ of $171 \pm 34 \mu \mathrm{M}$; in strong agreement with the $K_{D}$ of $154 \pm 30 \mu \mathrm{M}$ determined via $2 \mathrm{D}$ HSQC NMR titrations, with compound concentrations ranging between 0 and $2 \mathrm{mM}$.

Author Contributions: Conceptualisation, M.A., N.T.P. and S.S.; methodology, M.A., S.S. and N.T.P.; software, S.S.; validation, M.A., N.T.P. and S.S.; formal analysis, M.A., N.T.P., C.B.S., and S.S.; investigation, N.T.P. and S.S.; resources, M.A.; data curation, N.T.P. and S.S.; writing—original draft preparation, M.A., N.T.P., C.B.Ś. and S.S.; writing-review and editing, M.A., N.T.P., C.B.Ś. and S.S.; visualisation, N.T.P., C.B.Ś., and S.S.; supervision, M.A.; project administration, M.A.; funding acquisition, M.A. All authors have read and agreed to the published version of the manuscript.

Funding: The authors acknowledge financial support from the Scottish Universities Life Sciences Alliance (SULSAhttp://www.sulsa.ac.uk) and the Medical Research Council (MRC- www.mrc.ac.uk, J54359) Strategic Grant. M.A and N.T.P acknowledge financial support from the Wellcome Trust (Grant 201531/Z/16/Z).

Acknowledgments: The authors thank Ian Spink for collaborative work in the microdialysis of compound 4, and David Evans for discussion around microdialysis kinetics. In addition, we thank Logan Mackay and Van Kelly for their support with mass spectrometry experiments.

Conflicts of Interest: The authors declare no conflict of interest. The funders had no role in the design of the study; in the collection, analyses, or interpretation of data; in the writing of the manuscript, or in the decision to publish the results.

\section{References}

1. Nimmo, I.A.; Atkins, G.L.; Strange, R.C.; Percy-Robb, I.W. An evaluation of ways of using equilibrium dialysis to quantify the binding of ligand to macromolecules. Biochem. J. 1977, 165, 107-110. [CrossRef] [PubMed]

2. Zhou, A.; He, D.; Nie, L.H.; Yao, S.Z. Determination of the binding parameters of drug to protein by equilibrium dialysis/piezoelectric quartz crystal sensor. Anal. Biochem. 2000, 282, 10-15. [CrossRef] [PubMed]

3. Kariv, I.; Cao, H.; Oldenburg, K.R. Development of a high throughput equilibrium dialysis method. J. Pharm. Sci. 2001, 90, 580-587. [CrossRef]

4. Arkin, M.R.; Wells, J.A. Small-molecule inhibitors of protein-protein interactions: Progressing towards the dream. Nat. Rev. Drug Discov. 2004, 3, 301-317. [CrossRef] [PubMed]

5. Ward, L.D. Measurement of ligand binding to proteins by fluorescence spectroscopy. Methods Enzymol. 1985, 117, 400-414. [PubMed]

6. Meyer, B.; Peters, T. NMR spectroscopy techniques for screening and identifying ligand binding to protein receptors. Angew. Chem. Int. Ed. 2003, 42, 864-890. [CrossRef] [PubMed]

7. Höfner, G.; Wanner, K.T. Competitive binding assays made easy with a native marker and mass spectrometric quantification. Angew. Chem. Int. Ed. 2003, 42, 5235-5237. [CrossRef] [PubMed]

8. Annis, D.A.; Nickbarg, E.; Yang, X.; Ziebell, M.R.; Whitehurst, C.E. Affinity selection-mass spectrometry screening techniques for small molecule drug discovery. Curr. Opin. Chem. Biol. 2007, 11, 518-526. [CrossRef] [PubMed]

9. Loken, H.F.; Havel, R.J.; Gordan, G.S.; Whittington, S.L. Ultracentrifugal analysis of protein-bound and free calcium in human serum. J. Biol. Chem. 1960, 235, 3654-3658. [PubMed]

10. Zehender, H.; Le Goff, F.; Lehmann, N.; Filipuzzi, I.; Mayr, L.M. SpeedScreen: The "missing link" between genomics and lead discovery. J. Biomol. Screen. 2004, 9, 498-505. [CrossRef] [PubMed]

11. The Auer Lab. Affinity Selection. Available online: https://sites.google.com/view/the-auer-lab-uoe/screening/ affinity-selection (accessed on 28 June 2020).

12. Weidemann, T.; Seifert, J.-M.; Hintersteiner, M.; Auer, M. Analysis of protein-small molecule interactions by microscale equilibrium dialysis and its application as a secondary confirmation method for on-bead screening. J. Comb. Chem. 2010, 12, 647-654. [CrossRef] 
13. Waters, N.J.; Jones, R.; Williams, G.; Sohal, B. Validation of a rapid equilibrium dialysis approach for the measurement of plasma protein binding. J. Pharm. Sci. 2008, 97, 4586-4595. [CrossRef]

14. Van Liempd, S.; Morrison, D.; Sysmans, L.; Nelis, P.; Mortishire-Smith, R. Development and validation of a higher-throughput equilibrium dialysis assay for plasma protein binding. J. Assoc. Lab. Autom. 2011, 16, 56-67. [CrossRef] [PubMed]

15. Ye, Z.; Zetterberg, C.; Gao, H. Automation of plasma protein binding assay using rapid equilibrium dialysis device and Tecan workstation. J. Pharm. Biomed. Anal. 2017, 140, 210-214. [CrossRef] [PubMed]

16. Vanholder, R.; Hoefliger, N.; De Smet, R.; Ringoir, S.; Vogeleere, P. Extraction of protein bound ligands from azotemic sera: Comparison of 12 deproteinization methods. Kidney Int. 1992, 41, 1707-1712. [CrossRef] [PubMed]

17. Mathematica; Version 12.1; Wolfram Research, Inc.: Champaign, IL, USA, 2020.

18. Python Software Foundation. Python Language Reference, Version 3.8. Available online: http://www.python. org (accessed on 4 June 2020).

(C) 2020 by the authors. Licensee MDPI, Basel, Switzerland. This article is an open access article distributed under the terms and conditions of the Creative Commons Attribution (CC BY) license (http://creativecommons.org/licenses/by/4.0/). 